\title{
COMPLEMENTS OF INTERVALS AND PREFRATTINI SUBALGEBRAS OF SOLVABLE LIE ALGEBRAS
}

\author{
DAVID A. TOWERS
}

(Communicated by Kailash C. Misra)

\begin{abstract}
In this paper we study a Lie-theoretic analogue of a generalisation of the prefrattini subgroups introduced by W. Gaschütz. The approach follows that of P. Hauck and H. Kurzweil for groups by first considering complements in subalgebra intervals. Conjugacy of these subalgebras is established for a large class of solvable Lie algebras.
\end{abstract}

\section{Complements of subalgebra intervals}

Throughout, $L$ will denote a finite-dimensional solvable Lie algebra over a field $F$. For a subalgebra $U$ of $L$ we denote by $[U: L]$ the set of all subalgebras $S$ of $L$ with $U \subseteq S \subseteq L$. We say that $[U: L]$ is complemented if, for any $S \in[U: L]$, there is a $T \in[U: L]$ such that $S \cap T=U$ and $\langle S, T\rangle=L$, where $\langle S, T\rangle$ denotes the subalgebra of $L$ generated by $S$ and $T$. Our objective is to study the set

$$
\Omega(U, L)=\{S \in[U: L]:[S: L] \text { is complemented }\},
$$

in particular, to show that for a large class of solvable Lie algebras $L$, the minimal elements of this set, $\Omega(U, L)_{\text {min }}$, are conjugate in $L$. The development initially follows closely that of [3], but later the theory diverges from that for groups. For example, when $L^{2}$ is nilpotent, $\Omega(U, L)_{\min }$ contains just one element. When $L^{2}$ is not nilpotent $\Omega(U, L)_{\min }$ can contain more than one element but, unlike the grouptheoretic case, these elements may not be conjugate. In the second section these ideas are used to introduce the concept of prefrattini subalgebras of $L$; these were employed in [5] to study complemented solvable Lie algebras.

We denote by $[U: L]_{\max }$ the set of maximal subalgebras in $[U: L]$, that is, the set of maximal subalgebras of $L$ containing $U$. If $L=A+B$ where $A$ and $B$ are subalgebras of $L$ and $A \cap B=0$, we will write $L=A \oplus B$.

Lemma 1.1. If $S \in \Omega(U, L), S \neq L$, then $S=\bigcap\left\{M: M \in[S: L]_{\max }\right\}$.

Proof. Put $T=\bigcap\left\{M: M \in[S: L]_{\max }\right\}$. Then $[S: L]$ is complemented, since $S \in \Omega(U, L)$, and so $T$ has a complement $C$ in $[S: L]$. If $C \neq L$, then $C \subseteq M$ for some $M \in[S: L]_{\max }$. But then $\langle T, C\rangle \subseteq M$, contradicting the fact that $C$ is a complement of $T$ in $[S: L]$. Hence $C=L$ and $S=T \cap C=T \cap L=T$, as required.

Received by the editors September 9, 2011 and, in revised form, September 21, 2011.

2010 Mathematics Subject Classification. Primary 17B05, 17B30, 17 B50.

Key words and phrases. Lie algebras, complemented, solvable, Frattini ideal, prefrattini subalgebra, residual. 
The Frattini subalgebra of $L, \phi(L)$, is the intersection of the maximal subalgebras of $L$. When $L$ is solvable this is always an ideal of $L$, by [1, Lemma 3.4]. Extending this notion slightly we put $\phi(S, L)=\bigcap\left\{M: M \in[S: L]_{\max }\right\}$; clearly, $\phi(0, L)=$ $\phi(L)$. The above lemma shows that $\phi(U, L) \subseteq S$ for all $S \in \Omega(U, L)$.

Lemma 1.2. If $I$ is an ideal of $L$ and $S \in \Omega(U, L)$, then $S+I \in \Omega(U, L)$.

Proof. Let $B \in[S+I: L] \subseteq[S: L]$. Since $S \in \Omega(U, L), B$ has a complement $D$ in $[S: L]$; that is, $B \cap D=S$ and $\langle B, D\rangle=L$. Put $C=D+I$. Then $\langle B, C\rangle=L$ and $B \cap C=B \cap(D+I)=B \cap D+I=S+I$, whence $C$ is a complement for $B$ in $[S+I: L]$ and $S+I \in \Omega(U, L)$.

Lemma 1.3. Let $A$ be a minimal ideal of $L$ and let $M$ be a complement of $A$ in $L$ containing $U$. Then $\Omega(U, M)=\{S \in \Omega(U, L): S \subseteq M\}$. In particular $\Omega(U, M)_{\min }=\left\{S \in \Omega(U, L)_{\min }: S \subseteq M\right\}$.

Proof. Note that since $L$ is solvable, $M$ is a maximal subalgebra of $L$ and $L=$ $A \oplus M$. Suppose first that $S \in \Omega(U, L)$ with $S \subseteq M$. Then $S+A \in \Omega(U, L)$ by Lemma 1.2. The interval $[S: M]$ is lattice isomorphic to $[S+I: L]$ and so is complemented. Hence $S \in \Omega(U, M)$.

Conversely, let $S \in \Omega(U, M)$. Then $[S: M]$ is complemented. We need to show that $S \in \Omega(U, L)$, that is, that $[S: L]$ is complemented. Let $B \in[S: L]$. Then $B \cap M \in[S: M]$, so there is a subalgebra $D \in[S: M]$ such that $\langle B \cap M, D\rangle=M$ and $B \cap D=B \cap M \cap D=S$.

If $B \nsubseteq M$, then $M$ is a proper subalgebra of $\langle B, D\rangle$. But $M$ is a maximal subalgebra of $L$, and so $\langle B, D\rangle=L$ and $D$ is a complement of $B$ in $[S: L]$. Hence $[S: L]$ is complemented.

If $B \subseteq M$, put $C=D+A$. Then

$$
L=A \oplus M \subseteq\langle B, A\rangle+\langle B, D\rangle \subseteq\langle B, D+A\rangle=\langle B, C\rangle,
$$

so $\langle B, D+A\rangle=L$. Also

$$
B \cap C=B \cap(D+A)=B \cap M \cap(D+A)=B \cap(D+M \cap A)=B \cap D=S,
$$

yielding that $C$ is a complement of $B$ in $[S: L]$ and $[S: L]$ is complemented.

Lemma 1.4. Let $A$ be a minimal ideal of $L$ and let $S \in \Omega(U, L)_{\min }$ with $A \nsubseteq S$. Then there is an $M \in[S: L]_{\max }$ such that $A \nsubseteq M$.

Proof. This follows easily from Lemma 1.1

Lemma 1.5. Let $A$ be a minimal ideal of $L$. Then the following are equivalent:

(i) $A \nsubseteq S$ for some $S \in \Omega(U, L)_{\min }$;

(ii) $A \nsubseteq M$ for some $M \in[U: L]_{\max }$; and

(iii) for every $S \in \Omega(U, L)_{\text {min }}$ there is a complement of $A$ in $L$ containing $S$.

Proof. $(i) \Rightarrow$ (ii): This follows from Lemma 1.4.

(ii) $\Rightarrow($ iii $)$ : Suppose that $A \nsubseteq M$ for some $M \in[U: L]_{\max }$. Then $L=A \oplus M$. Let $S \in \Omega(U, L)_{\min }$.

Suppose first that $A \subseteq S$. Then $S=A \oplus M \cap S$ and $M \cap S \cong S / A$, so the interval $[S: L]$ is lattice isomorphic to $[M \cap S: M]$. It follows that $M \cap S \in \Omega(U, M)$. But Lemma 1.3 now gives that $M \cap S \in \Omega(U, L)$, contradicting the minimality of $S$.

Hence $A \nsubseteq S$ and Lemma 1.4 gives a complement of $A$ containing $S$.

$($ iii $) \Rightarrow(i)$ : This is trivial. 
Lemma 1.6. If $A$ is an ideal of $L$ and $S \in \Omega(U, L)_{\text {min }}$, then

$$
S+A \in \Omega(U+A, L)_{\min } .
$$

Proof. It suffices to show that $(S+A) / A \in \Omega((U+A) / A, L / A)_{\min }$ and so we may suppose that $A$ is a minimal ideal of $L$. The result is clear if $A \subseteq S$, since then $U+A \subseteq S$. So suppose that $A \nsubseteq \subseteq$.

Then there is a complement $M$ of $A$ in $L$ containing $S$, by Lemma 1.5] and $L=A \oplus M$. Moreover, $S+A \in \Omega(U+A, L)$. Choose $C \in \Omega(U+A, L)_{\text {min }}$ such that $C \subseteq S+A$. Then $U \subseteq M \cap C \subseteq S \subseteq M$ and the interval $[M \cap C: M]$ is lattice isomorphic to $[C: L]$. It follows that $M \cap C \in \Omega(U, M)$ and so $M \cap C \in \Omega(U, L)$, by Lemma 1.3. But $S \in \Omega(U, L)_{\text {min }}$, which yields that $M \cap C=S$; that is, $C=S+A$.

At this point the theory starts to diverge from that for groups. We say that $L$ is completely solvable if $L^{2}$ is nilpotent. For these algebras $\Omega(U, L)_{\text {min }}$ takes on a particularly simple form.

Theorem 1.7. Let $L$ be completely solvable and let $U$ be a subalgebra of $L$. Then $\Omega(U, L)_{\min }=\{\phi(U, L)\}$. In particular, if $U=0$, then $\Omega(U, L)_{\min }=\{\phi(L)\}$.

Proof. Let $B \in \Omega(U, L)_{\min }, C=\phi(U, L)$. Then $\phi(U, L) \subseteq B$ and so $C \subseteq B$, by Lemma 1.1. We now use induction on the dimension of $L$. Suppose first that there is a minimal ideal $A$ of $L$ with $A \subseteq C$. Then $B / A \in \Omega((U+A) / A, L / A)_{\text {min }}$, by Lemma 1.6, and so $B / A=\phi((U+A) / A, L / A)$, by the inductive hypothesis. From this it is clear that $B=C$.

So suppose now that no such minimal ideal exists. Then $\phi(L)=0$ and so $L$ is complemented, by [4, Theorem 1]. Thus there is a subalgebra $V$ such that $\langle C, V\rangle=L$ and $C \cap V=0$. It follows that $\langle C, U+V\rangle=L$ and $C \cap(U+V)=$ $U+C \cap V=U$, whence $C \in[U: L]$ and $[C: L]$ is complemented. Thus $C \in \Omega(U, L)$ and the minimality of $B$ yields that $B=C$.

If $L$ is not completely solvable, then $\Omega(U, L)_{\min }$ can contain more than one element as we shall see in the next section. However, we do have a conjugacy result in some cases. First we need to consider inner automorphisms of $L$. Let $x \in L$ and let ad $x$ be the corresponding inner derivation of $L$. If $F$ has characteristic zero, suppose that $(\operatorname{ad} x)^{n}=0$ for some $n$; if $F$ has characteristic $p$, suppose that $x \in I$, where $I$ is a nilpotent ideal of $L$ of class less than $p$. Put

$$
\exp (\operatorname{ad} x)=\sum_{r=0}^{\infty} \frac{1}{r !}(\operatorname{ad} x)^{r} .
$$

Then $\exp (\operatorname{ad} x)$ is an automorphism of $L$. We shall call the group $\mathcal{I}(L)$ generated by all such automorphisms the group of inner automorphisms of $L$. More generally, if $B$ is a subalgebra of $L$ we denote by $\mathcal{I}(L: B)$ the group of automorphisms of $L$ generated by the $\exp (\operatorname{ad} x)$ with $x \in B$.

If $B$ is a subalgebra of $L$, the centraliser of $B$ in $L$ is $C_{L}(B)=\{x \in L$ : $[x, B]=0\}$. We define the nilpotent residual to be $L^{\infty}=\bigcap_{i=1}^{\infty} L^{i}$, where the $L^{i}$ are the terms of the lower central series for $L$. Then we have conjugacy for the following metanilpotent Lie algebras. 
Theorem 1.8. Suppose that $L$ is a solvable Lie algebra over a field $F$ of characteristic $p$, and suppose further that $L^{\infty}$ has nilpotency class less than $p$. Let $U$ be a subalgebra of $L$. Then the elements of $\Omega(U, L)_{\text {min }}$ are conjugate under $\mathcal{I}\left(L: L^{\infty}\right)$.

Proof. We use induction on the dimension of $L$. It is clearly true if $L$ has dimension one, so suppose it holds for such algebras with dimension smaller than that of $L$. We can assume that $L^{\infty} \neq 0$. Let $S_{1}, S_{2} \in \Omega(U, L)_{\text {min }}$ and let $A$ be a minimal ideal of $L$ with $A \subseteq L^{\infty}$. Then $\left(S_{1}+A\right) / A,\left(S_{2}+A\right) / A \in \Omega((U+A) / A, L / A)_{\text {min }}$, by Lemma 1.6. and so $\left(S_{1}+A\right) / A$ and $\left(S_{2}+A\right) / A$ are conjugate under $\mathcal{I}\left(L / A: L^{\infty} / A\right)$, by the inductive hypothesis.

If $A \subseteq S_{1}$, then $A \subseteq S_{2}$, by Lemma 1.5, and there is an $x \in L^{\infty}$ such that $S_{1} \exp (\operatorname{ad} x)=S_{2}$; that is, $S_{1}$ and $S_{2}$ are conjugate under $\mathcal{I}\left(L: L^{\infty}\right)$.

So suppose that $A \nsubseteq S_{1}$. Then there are complements $M_{1}$ and $M_{2}$ of $A$ in $L$ with $S_{1} \subseteq M_{1}$ and $S_{2} \subseteq M_{2}$, by Lemma 1.5. Put $C=C_{M_{1}}(A)$, which is an ideal of $L$. If $C=0$, then $C_{L}(A)=A$ and there is $a \in A$ such that $M_{2} \exp (\operatorname{ad} a)=M_{1}$, by [2, Theorem 1.1], whence $S_{2} \exp (\operatorname{ad} a) \subseteq M_{2} \exp (\operatorname{ad} a)=M_{1}$.

If $C \neq 0$, then $\left(S_{1}+C\right) / C$ and $\left(S_{2}+C\right) / C$ are conjugate under $\mathcal{I}(L / C$ : $\left.\left(L^{\infty}+C\right) / C\right)$, by the inductive hypothesis. It follows that there is an $x \in L^{\infty}$ such that $S_{2} \exp (\operatorname{ad} x+C) \subseteq S_{1}+C \exp (\operatorname{ad} a) \subseteq M_{1}$, which gives $S_{2} \exp (\operatorname{ad} x) \subseteq M_{1}$. Now $L=A \oplus M_{1}$, so $L^{\infty} \subseteq A \oplus M_{1}^{\infty}$. Moreover, $\left[A, L^{\infty}\right]=0$ since $L^{\infty}$ is nilpotent, so $M_{1}^{\infty}$ is an ideal of $L$. Put $x=a+b$, where $a \in A, b \in M_{1}^{\infty}$. Then, for each $s_{2} \in S_{2}$, we have $s_{2}+s_{2}$ ad $x+\ldots+s_{2}(\operatorname{ad} x)^{n} \in M_{1}$, which gives $s_{2}+s_{2}$ ad $a \in M_{1}$. Thus, again we have that $S_{2} \exp (\operatorname{ad} a) \subseteq M_{1}$ for some $a \in A$.

So $S_{1}, S_{2} \exp (\operatorname{ad} a) \subseteq M_{1}$ for some $a \in A$. Now $U \subseteq S_{1} \subseteq M_{1}$ and $U \exp (\operatorname{ad} a) \subseteq S_{2} \exp (\operatorname{ad} a) \subseteq M_{1}$, so, for each $u \in U, u+[a, u] \in M_{1}$, which gives $[a, u] \in A \cap M_{1}=0$; that is, $a \in C_{L}(U)$ and $U \exp (\operatorname{ad} a)=U$. Thus

$$
S_{2} \exp (\operatorname{ad} a) \in \Omega(U \exp (\operatorname{ad} a), L)_{\min }=\Omega(U, L)_{\min } .
$$

But now Lemma 1.3 yields that $S_{1}, S_{2} \exp (\operatorname{ad} a) \in \Omega\left(U, M_{1}\right)_{\text {min }}$, and the required conjugacy of $S_{1}$ and $S_{2}$ follows from the inductive hypothesis.

\section{2. $U$-PREFRATTINI SUBALGEBRAS}

Let

$$
0=A_{0} \subset A_{1} \subset \ldots \subset A_{n}=L
$$

be a fixed chief series for $L$. We say that $A_{i} / A_{i-1}$ is a Frattini chief factor if $A_{i} / A_{i-1} \subseteq \phi\left(L / A_{i-1}\right)$; it is complemented if there is a maximal subalgebra $M$ of $L$ such that $L=A_{i}+M$ and $A_{i} \cap M=A_{i-1}$. When $L$ is solvable it is easy to see that a chief factor is Frattini if and only if it is not complemented. This can be generalised as follows.

The factor algebra $A_{i} / A_{i-1}$ is called a $U$-Frattini chief factor if

$$
A_{i} \subseteq \phi\left(U+A_{i-1}, L\right) \text { or if } U+A_{i-1}=L,
$$

that is, if every maximal subalgebra of $L$ which contains $U$ and $A_{i-1}$ also contains $A_{i}$. If $A_{i} / A_{i-1}$ is not a $U$-Frattini chief factor there is an $M \in\left[U+A_{i-1}: L\right]_{\max }$

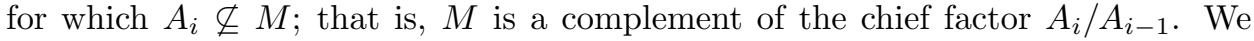
have a sharpened form of the Jordan-Hölder Theorem in which the $U$-Frattini chief factors correspond. First we need a lemma. 
Lemma 2.1. Let $A_{1}, A_{2}$ be distinct minimal ideals of the solvable Lie algebra $L$. Then there is a bijection

$$
\theta:\left\{A_{1},\left(A_{1}+A_{2}\right) / A_{1}\right\} \rightarrow\left\{A_{2},\left(A_{1}+A_{2}\right) / A_{2}\right\}
$$

such that corresponding chief factors are isomorphic as L-modules and U-Frattini chief factors correspond to one another.

Proof. Clearly we can assume that $U \neq L$. Put $A=A_{1} \oplus A_{2}$. Suppose first that $A_{1}$ is a $U$-Frattini chief factor. Then $A_{1} \subseteq \phi(U, L)$. Thus $A \subseteq \phi\left(U+A_{2}, L\right)$ and $A / A_{2}$ is a $U$-Frattini chief factor. If $A / A_{1}$ is also a $U$-Frattini chief factor, then $A \subseteq \phi\left(U+A_{1}, L\right)$, which yields that $A \subseteq \phi(U, L)$, and all four factors are $U$-Frattini. In this case we can choose $\theta$ so that $\theta\left(A_{1}\right)=A / A_{2}$ and $\theta\left(A / A_{1}\right)=A_{2}$. If $A / A_{1}$ is not a $U$-Frattini chief factor, then neither is $A_{2}$, by the same argument as above, and so the same choice of $\theta$ suffices and likewise if none of the factors are $U$-Frattini chief factors.

The remaining case is where $A_{1}$ and $A_{2}$ are not $U$-Frattini chief factors but $A / A_{2}$ is. The fact that $A / A_{2}$ is a $U$-Frattini chief factor means that every maximal subalgebra containing $U$ and $A_{2}$ also contains $A$, and so contains $A_{1}$. But, since $A_{1}$ is not a $U$-Frattini chief factor, there is a maximal subalgebra $M$ containing $U$ but not containing $A_{1}$. It follows that $A_{2} \nsubseteq M$. Thus $M$ complements both $A_{1}$ and $A_{2}$ in $L$. Put $C=A \cap M$. Then $L / A_{i} \cong M$ and this isomorphism maps the set of maximal subalgebras of $L / A_{i}$ which contain $\left(U+A_{i}\right) / A_{i}$ onto the set of maximal subalgebras of $M$ which contain $U$. Since $A / A_{2}$ is a $U$-Frattini chief factor, every maximal subalgebra of $L$ containing $U+A_{2}$ contains $A$, so every maximal subalgebra of $M$ which contains $U$ also contains $C$. It follows that every maximal subalgebra of $L$ which contains $U+A_{1}$ also contains $C+A_{1}$; that is, $A / A_{1}$ is a $U$-Frattini chief factor of $L$. So we can choose $\theta$ so that $\theta\left(A_{1}\right)=A_{2}$ and $\theta\left(A / A_{1}\right)=A / A_{2}$.

Theorem 2.2. Let

$$
\begin{gathered}
0<A_{1}<\ldots<A_{n}=L, \\
0<B_{1}<\ldots<B_{n}=L
\end{gathered}
$$

be chief series for the solvable Lie algebra L. Then there is a bijection between the chief factors of these two series such that corresponding factors are isomorphic as $L$-modules and such that the U-Frattini chief factors in the two series correspond.

Proof. These two series have the same length by a version of the Jordan-Hölder Theorem. We use induction on $n$. The result is clearly true if $n=1$. So let $n>1$ and suppose that the result holds for all solvable Lie algebras with chief series of length $\leq n-1$. If $A_{1}=B_{1}$, then applying the inductive hypothesis to $L / A_{1}$ gives a suitable bijection between the factors above $A_{1}$, and then we can map $A_{1}$ to $B_{1}$ and we have the result.

So suppose that $A_{1}$ and $B_{1}$ are distinct and put $A=A_{1} \oplus B_{1}$. Then $A / A_{1}$ and $A / B_{1}$ are chief factors of $L$ and there are chief series of the form

$$
\begin{aligned}
& 0<A_{1}<A<C_{3}<\ldots<C_{n}=L, \\
& 0<B_{1}<A<C_{3}<\ldots<C_{n}=L .
\end{aligned}
$$

Define an equivalence relation on the chief series of $L$ by saying that two such series are equivalent if there is a bijection between their chief factors satisfying 
the requirements of the theorem. Since series (2) and (4) have a minimal ideal in common, they are equivalent. Similarly, series (3) and (5) are equivalent. Moreover, since series (4) and (5) coincide above $A$ they are also equivalent, by Lemma 2.1 . Hence the series (2) and (3) are equivalent, as required.

We define the set $\mathcal{I}$ by $i \in \mathcal{I}$ if and only if $A_{i} / A_{i-1}$ is not a $U$-Frattini chief factor of $L$. For each $i \in \mathcal{I}$ put

$$
\mathcal{M}_{i}=\left\{M \in\left[U+A_{i-1}, L\right]_{\max }: A_{i} \nsubseteq \mathbb{M}\right\} .
$$

Then $B$ is a $U$-prefrattini subalgebra of $L$ if

$$
B=\bigcap_{i \in \mathcal{I}} M_{i} \text { for some } M_{i} \in \mathcal{M}_{i}
$$

If $U=0$ we will refer to $B$ simply as a prefrattini subalgebra of $L$.

The subalgebra $B$ avoids $A_{i} / A_{i-1}$ if $B \cap A_{i}=B \cap A_{i-1}$; likewise, $B$ covers $A_{i} / A_{i-1}$ if $B+A_{i}=B+A_{i-1}$. Then we have the following important property of $U$-prefrattini subalgebras of $L$.

Lemma 2.3. If $B$ is a $U$-prefrattini subalgebra of $L$, then it covers all $U$-Frattini chief factors of $L$ in (1) and avoids the rest.

Proof. Let $B$ be a $U$-prefrattini subalgebra of $L$ and let $A_{i} / A_{i-1}$ be a chief factor of $L$. If it is a $U$-Frattini chief factor, then either $A_{i} \subseteq \phi\left(U+A_{i-1}, L\right)$ or else $U+A_{i-1}=L$. In the former case, every maximal subalgebra of $L$ that contains $U+A_{i-1}$ also contains $A_{i}$, and so $A_{i} \subseteq B$. In either case, therefore, $B$ covers $A_{i} / A_{i-1}$. If it is not a $U$-Frattini chief factor we have $B \subseteq M_{i}$ where $L=A_{i}+M_{i}$ and $A_{i} \cap M_{i}=A_{i-1}$. Hence $B \cap A_{i}=B \cap M_{i} \cap A_{i}=B \cap A_{i-1} \subseteq B \cap A_{i}$, and so $B$ avoids $A_{i} / A_{i-1}$.

The next four results are dedicated to showing how the $U$-prefrattini subalgebras relate to the material in the previous section. The first lemma is helpful when trying to calculate $U$-prefrattini subalgebras.

Lemma 2.4. Let $B$ be a $U$-prefrattini subalgebra of $L$. Then

$$
\operatorname{dim} B=\sum_{i \notin \mathcal{I}}\left(\operatorname{dim} A_{i}-\operatorname{dim} A_{i-1}\right) ;
$$

in particular, all $U$-prefrattini subalgebras of $L$ have the same dimension.

Proof. We use induction on $\operatorname{dim} L$. The result is clear if $L$ is abelian, so suppose it holds for Lie algebras of smaller dimension than $L$. It is easy to check that $\left(B+A_{1}\right) / A_{1}$ is a $\left(\left(U+A_{1}\right) / A_{1}\right)$-prefrattini subalgebra of $L / A_{1}$ and so

$$
\operatorname{dim}\left(\frac{B+A_{1}}{A_{1}}\right)=\sum_{i \in I, i \neq 1}\left(\operatorname{dim} A_{i}-\operatorname{dim} A_{i-1}\right),
$$

by the inductive hypothesis. If $A_{1} / A_{0}$ is a $U$-Frattini chief factor of $L$, then $B$ covers $A_{1} / A_{0}$, whence $B=B+A_{1}$ and

$$
\operatorname{dim} B=\operatorname{dim} A_{1}+\operatorname{dim}\left(\frac{B+A_{1}}{A_{1}}\right)=\sum_{i \in I}\left(\operatorname{dim} A_{i}-\operatorname{dim} A_{i-1}\right) .
$$


If $A_{1} / A_{0}$ is not a $U$-Frattini chief factor of $L$, then $B$ avoids $A_{1} / A_{0}$, whence $B \cap A_{1}=$ 0 and

$$
\operatorname{dim} B=\operatorname{dim}\left(\frac{B+A_{1}}{A_{1}}\right)=\sum_{i \in I}\left(\operatorname{dim} A_{i}-\operatorname{dim} A_{i-1}\right)
$$

Let $\Pi(U, L)$ be the set of $U$-prefrattini subalgebras of $L$.

Lemma 2.5. $\Pi(U, L) \subseteq \Omega(U, L)$.

Proof. (i) We use induction on $\operatorname{dim} L$. The result is clear if $L$ is abelian, so suppose it holds for Lie algebras of dimension less than that of $L$. Let $B \in \Pi(U, L)$. Then

$$
\frac{B+A_{1}}{A_{1}} \in \Pi\left(\frac{U+A_{1}}{A_{1}}, \frac{L}{A_{1}}\right) \subseteq \Omega\left(\frac{U+A_{1}}{A_{1}}, \frac{L}{A_{1}}\right),
$$

whence $B+A_{1} \in \Omega(U, L)$. If $A_{1} \subseteq B$ we have $B \in \Omega(U, L)$. So suppose that $A_{1} \not \subset B$. Then $B$ does not cover $A_{1} / A_{0}$, so $A_{1} / A_{0}$ is not a $U$-Frattini chief factor of $L$. It follows that $1 \in \mathcal{I}$, and so there is a maximal subalgebra $M$ of $L$ with $B \subseteq M$ and $A_{1} \nsubseteq M$. But now $L=A_{1} \oplus M$ and the intervals $\left[B+A_{1}: L\right]$ and $[B: M]$ are lattice isomorphic, which yields that $[B: M]$ is complemented. It follows from Lemma 1.3 that $B \in \Omega(U, L)$ again.

Lemma 2.6. $\Omega(U, L)_{\min } \subseteq \Pi(U, L)$.

Proof. Let $B \in \Omega(U, L)_{\min }$ and let $A_{i} / A_{i-1}$ be a chief factor of $L$. By Lemma 1.6.

$$
\left(\frac{B+A_{i-1}}{A_{i-1}}\right) \in \Omega\left(\frac{U+A_{i-1}}{A_{i-1}}, \frac{L}{A_{i-1}}\right)_{\min } .
$$

We now apply Lemma 1.5 to the minimal ideal $A_{i} / A_{i-1}$ of $L / A_{i-1}$. If $A_{i} / A_{i-1}$ is a $U$-Frattini chief factor, then it does not have a complement in $L / A_{i-1}$ and Lemma 1.5 gives that $A_{i} \subseteq B+A_{i-1}$, whence $A_{i}+B=A_{i-1}+B$ and $B$ covers $A_{i} / A_{i-1}$.

If $A_{i} / A_{i-1}$ is not a $U$-Frattini chief factor, then it has a complement $M_{i} / A_{i-1}$ in $L / A_{i-1}$ and Lemma [1.5] gives that it has such a complement containing $\left(B+A_{i-1}\right) / A_{i-1}$; that is, $L=M_{i}+A_{i}, M_{i} \cap A_{i}=A_{i-1}$ and $B+A_{i-1} \subseteq M_{i}$. But now $B \cap A_{i} \subseteq B \cap A_{i}+A_{i-1}=\left(B+A_{i-1}\right) \cap A_{i} \subseteq M_{i} \cap A_{i}=A_{i-1}$. It follows that $B \cap A_{i}=B \cap A_{i-1}$ and $B$ avoids $A_{i} / A_{i-1}$. Clearly $M_{i} \in \mathcal{M}_{i}$ and $B \subseteq C=\bigcap_{i \in \mathcal{I}} M_{i} \in \Pi(U, L)$. But $B$ covers or avoids the same chief factors of (1) as $C$, so the proof of Lemma 2.4 shows that $\operatorname{dim} B=\operatorname{dim} C$. It follows that $B=C \in \Pi(U, L)$.

Putting the previous three lemmas together yields the following result.

Theorem 2.7. $\Omega(U, L)_{\min }=\Pi(U, L)$.

Notice that, in particular, the above result shows that the definition of $U$ prefrattini subalgebras does not depend on the choice of chief series.

Corollary 2.8. If $A$ is an ideal of $L$ and $S \in \Pi(U, L)$, then

$$
(S+A) / A \in \Pi((U+A) / A, L / A) .
$$

Proof. This follows from Theorem 2.7 and Lemma 1.6 
Corollary 2.9. For every solvable Lie algebra $L$,

$$
\phi(U, L)=\bigcap_{B \in \Pi(U, L)} B .
$$

Proof. Put $P=\bigcap_{B \in \Pi(U, L)} B$. Then $\phi(U, L) \subseteq P$, by Theorem 2.7 and Lemma 1.1. Let $M \in[U, L]_{\max }$. There is an $i$ such that $A_{i-1} \subseteq M$ but $A_{i} \not \subset(1 \leq i \leq n)$. Then $A_{i} / A_{i-1}$ is not a $U$-Frattini chief factor of $L$, so $i \in \mathcal{I}$ and $M \in \mathcal{M}_{i}$. Thus there is $B \in \Pi(U, L)$ such that $B \subseteq M$, whence $P \subseteq M$. Hence $P \subseteq \phi(U, L)$.

Corollary 2.10. Let $L$ be completely solvable and let $U$ be a subalgebra of $L$. Then $\Pi(U, L)=\{\phi(U, L)\}$. In particular, $\Pi(0, L)=\{\phi(L)\}$.

Proof. This follows from Theorem 2.7 and Theorem 1.7 .

Corollary 2.11. Suppose that $L$ is a solvable Lie algebra over a field $F$ of characteristic $p$, and suppose further that $L^{\infty}$ has nilpotency class less than $p$. Let $U$ be a subalgebra of $L$. Then the elements of $\Pi(U, L)$ are conjugate under $\mathcal{I}\left(L: L^{\infty}\right)$.

Proof. This follows from Theorem 2.7 and Theorem 1.8 .

If $L^{2}$ is not nilpotent, then $\Pi(U, L)$ can contain more than one element, as the following example shows.

Example 2.1. Let $F$ be a field of characteristic $p$ (perfect if $p=2$ ), and $L=$ $\left(\bigoplus_{i=0}^{p-1} F e_{i}\right) \oplus F c \oplus F s \oplus F x$ with $\left[e_{i}, c\right]=e_{i},\left[e_{i}, s\right]=e_{i+1}$ for $i=0, \ldots, p-2$, $\left[e_{p-1}, s\right]=0,\left[e_{i}, x\right]=i e_{i-1}$ for $i=0, \ldots, p-1$ and $e_{-1}=0,[s, x]=c$, and all other products are zero.

$$
\begin{gathered}
\text { Put } A_{0}=0, A_{1}=\bigoplus_{i=0}^{p-1} F e_{i}, A_{2}=A_{1} \oplus F c, A_{3}=A_{2} \oplus F s, A_{4}=L \text {. Then } \\
0=A_{0} \subset A_{1} \subset A_{2} \subset A_{3} \subset A_{4}=L
\end{gathered}
$$

is a chief series for $L$ in which $A_{2} / A_{1}$ is the only Frattini chief factor. It is, therefore, straightforward to see that the prefrattini subalgebras of $L$ are the one-dimensional subalgebras $F(\alpha c+a)$, where $a \in A_{1}=L^{\infty}, \alpha \in F$.

Note that these are all conjugate under inner automorphisms of the form $1+$ ad $a$. This is not always the case, however. For, if $B$ is a faithful irreducible $L$ module and we form $X=B \oplus L$, where $B^{2}=0$ and $L$ acts on $B$ under the given $L$-module action, then the prefrattini subalgebras are still of the form $F(\alpha c+a)$ where $a \in A_{1}$. However, $B$ is the unique minimal ideal of $L$ and these subalgebras are not conjugate under inner automorphisms of the form $1+\operatorname{ad} b, b \in B$. Since $B$ is the nilradical of $X$, defining other inner automorphisms is problematic. Note that $X^{\infty}=B+A_{1}$, which is not nilpotent.

\section{ACKNOWLEDGEMENT}

The author is grateful to the referee for correcting an error in the original version of Lemma 2.1.

\section{REFERENCES}

[1] D.W. Barnes and H.M. Gastineau-Hills, 'On the theory of soluble Lie algebras', Math. Z. 106 (1968), 343-354. MR0232807 (38:1130)

[2] D.W. BARnes And M.L. Newell, 'Some theorems on saturated homomorphs of soluble Lie algebras', Math. Z. 115 (1970), 179-187. MR0266969(42:1871) 
[3] P. Hauck And H. Kurzweil, 'A lattice-theoretic characterization of prefrattini subgroups', Manuscripta Math. 66 (1990), 295-301. MR.1031198 (91f:20026)

[4] D.A. Towers, 'On complemented Lie algebras', J. London Math. Soc. (2) 22 (1980), 63-65. MR.579809 (81h:17006)

[5] D. A. Towers, 'Solvable complemented Lie algebras', Proc. Amer. Math. Soc. 140 (2012), no. 11, 3823-3830. MR2944723

Department of Mathematics and Statistics, Lancaster University, Lancaster LA1 $4 \mathrm{YF}$, ENGLAND

E-mail address: d.towers@lancaster.ac.uk 